Article

\title{
Preparation of Magnetic Iron Oxide Nanoparticles (MIONs) with Improved Saturation Magnetization Using Multifunctional Polymer Ligand
}

\author{
Muhammad Irfan Majeed ${ }^{1,+}+$, Jiaojiao Guo ${ }^{1,2,+}$, Wei Yan ${ }^{1,2, *}$ and Bien Tan ${ }^{1}$ \\ 1 School of Chemistry and Chemical Engineering, Huazhong University of Science and Technology, \\ Wuhan 430074, China; irfanmajeed2003@gmail.com (M.I.M.); gij824405637@gmail.com (J.G.); \\ bien.tan@mail.hust.edu.cn (B.T.) \\ 2 Hubei Collaborative Innovation Center for Advanced Organic Chemical Materials, Ministry of Education, \\ Key Laboratory of Green Preparation and Application for Functional Materials, Hubei Key Laboratory of \\ Polymer Materials, School of Materials Science \& Engineering, Hubei University, Wuhan 430062, China \\ * Correspondence: willieyancn2003@aliyun.com; Tel.: +86-27-8755-8172 \\ $\dagger$ These authors contributed equally to this work. \\ $\ddagger$ Current address: Department of Chemistry, University of Agriculture Faisalabad, \\ Faisalabad 38040, Pakistan.
}

Academic Editors: Joannis K. Kallitsis, Georgios Bokias and Valadoula Deimede Received: 4 October 2016; Accepted: 31 October 2016; Published: 8 November 2016

\begin{abstract}
This paper describes the preparation of ultra-small magnetic iron oxide $\left(\mathrm{Fe}_{3} \mathrm{O}_{4}\right)$ nanoparticles (MIONs) coated with water-soluble thioether end-functionalized polymer ligand pentaerythritol tetrakis 3-mercaptopropionate-polymethacrylic acid (PTMP-PMAA). The MIONs were prepared by co-precipitation of aqueous iron precursor solution at a high temperature. The polymer modified MIONs were characterized by dynamic light scattering (DLS), transmission electron microscopy (TEM), Fourier transform infrared spectroscopy (FTIR), X-ray powder diffraction (XRD), thermogravimetric analysis (TGA), and vibrating sample magnetometery (VSM). It was found that these MIONs were successfully modified by this water-soluble polymer ligand with a fairly uniform size and narrow size distribution. The dried powder of MIONs could be stored for a long time and re-dispersed well in water without any significant change. Additionally, the polymer concentration showed a significant effect on size and magnetic properties of the MIONs. The saturation magnetization was increased by optimizing the polymer concentration. Furthermore, the 3-(4,5-dimethylthiazol-2-yl)-2-5-diphenyltetrazolium bromide (MTT)-assay demonstrated that these MIONs were highly biocompatible and they could be successfully coupled with fluorescent dye Rhodamine due to the formation of amide bond between carboxylic acid groups of MIONs and amine groups of dye. The obtained results indicated that these multifunctional MIONs with rich surface chemistry exhibit admirable potential in biomedical applications.
\end{abstract}

Keywords: magnetic iron oxide nanoparticles; polymer ligand; biocompatible; multifunctional; saturation magnetization

\section{Introduction}

Magnetic nanoparticles (NPs) have gained much scientific interest for their unique magnetic properties such as superparamagnetism, high coercivity, low Curie temperature, and high magnetic susceptibility [1,2]. Magnetic iron oxide nanoparticles (MIONs), owing to their advantages such as low toxicity and biocompatibility, are considered to be the most favorable candidates for bio-applications [3] including magnetic resonance imaging (MRI) [4,5], magnetic fluid hyperthermia (MFH) [6,7], magnetic separation and immobilization of biomolecules such as nucleic acids and proteins $[8,9]$, 
the development of drug delivery systems for controlled release of drugs [10,11], biolabeling and magnetic sensors [12]. Thermal decomposition and co-precipitation are among the most common techniques employed for MIONs preparation but both having advantages and limitations [13]. It is usually desired to develop a simple, one step, cost effective and environment friendly protocol for the preparation of water-soluble, uniform, multifunctional, superparamagnetic iron oxide NPs with a better control over their size, shape and magnetic properties. Furthermore, for bioapplications of MIONs, they should be highly dispersed in aqueous phase and biocompatible having least or no toxicity, which can be achieved through adopting co-precipitation method using multifunctional water-soluble polymers as capping ligands.

Multifunctional water-soluble polymers as capping ligands can play an important role in the preparation of MIONs and other inorganic NPs [14-17]. In aqueous co-precipitation process, polymer ligands can efficiently control the size and shape of the NPs due to the presence of abundant functional groups such as $-\mathrm{COOH},-\mathrm{OH},-\mathrm{NH}_{2}$, etc. These multifunctional polymers render magnetic NPs more stable, water-soluble and uniform in addition to providing them rich surface chemistry which opens up ways for easier post-synthesis modification and functionalization for bio-applications [5,18-22]. Numerous studies have been reported for the synthesis of MIONs using polymer ligands in order to render them water-soluble and biocompatible. However, in most of the cases, the NPs are first prepared through thermal decomposition and then made water-soluble through post-synthesis modification processes such as ligand exchange using water-soluble polymer ligands [23,24].

In this study, we have described the synthesis of MIONs with a multifunctional water-soluble polymer ligand pentaerythritol tetrakis 3-mercaptopropionate-polymethacrylic acid (PTMP-PMAA), following our previous work [20]. Previously, it was demonstrated that PTMP-PMAA, having abundance of carboxylic acid groups, can be successfully used for the stabilization of the MIONs in the co-precipitation procedure. These carboxylic acid groups have ability to cap the growing MIONs in the reaction mixture through coordinating with iron oxide surface and thus stabilize them and control their size and size distribution depending upon the concentration of the polymer ligand. Therefore, the concentration of the polymer ligand or the molar ratio between carboxylic acid groups of the PTMP-PMAA and iron precursors play an important role in preparation of uniform MIONs through aqueous co-precipitation procedure. However, in that report, MIONs prepared with $0.768 \mathrm{mM}$ concentration of PTMP-PMAA had very small size $(4.5 \pm 0.4 \mathrm{~nm})$ and lower saturation magnetization $\left(45 \mathrm{emu} \cdot \mathrm{g}^{-1}\right)$. Those MIONs, due to their high dispersibility and ultra-small size, were successfully used as dual MRI contrast agents [5]. However, due to their lower saturation magnetization, they could not be manipulated in dispersed state with the use of an external magnet, which limits their scope of applications.

Therefore, herein, we report the preparation of MIONs using PTMP-PMAA with improved saturation magnetization through our original high temperature single step co-precipitation method [20] but with few modifications in order to improve the magnetic properties of MIONs, such as lower polymer concentrations as compared to previous report, iron precursors were dissolved in concentrated hydrochloric acid $(\mathrm{HCl})$ instead of $1 \mathrm{M} \mathrm{HCl}$ solution in order to prevent their hydrolysis and condensation before the addition of precipitating agents and the inert conditions were maintained, by nitrogen gas bubbling throughout the course of reaction, which not only protects MIONs from critical oxidation but also keeps their size smaller [25].

MIONs prepared using PTMP-PMAA had several carboxylic acid (-COOH) functional groups which provide them excellent dispersibility and stability in aqueous solutions. These MIONs showed high resistance against aggregation in aqueous media over a wide range of $\mathrm{pH}$ and salt concentration due to excellent electrostatic and steric stabilization provided by the polymer ligand. These MIONs can be dried by evaporating solvent and stored as powder for several months without any undesired changes in their chemical and physical properties. The polymer ligand offers MIONs better chemical stability against oxidation which otherwise leads to a decrease in their magnetic properties. Furthermore, cytotoxicity analysis of the MIONs proved them to be biocompatible even at their high 
concentration up to $500 \mu \mathrm{g} \cdot \mathrm{mL}^{-1}$. Bio-applicability of these NPs was demonstrated by successfully conjugation of MIONs with a fluorescent dye Rhodamine (Rh 110). Such bimodal detection systems based on fluorescent molecules and magnetic NPs can facilitate the deep tissue imaging by combined optical and MRI techniques [26]. The fabrication of novel targeted luminescent and magnetic NPs with multifunctional water-soluble polymers would play a vital role in the development of contrast agents for diagnosis, imaging and therapeutic technologies of the new era [26,27]. Finally, it was concluded that MIONs stabilized with PTMP-PMAA were extremely biocompatible and can be used for several bio-applications due to their chemically rich surface providing numerous opportunities for their conjugation with a variety of therapeutic, targeting, and labeling agents.

\section{Materials and Methods}

\subsection{Materials}

All chemicals were of analytical grade and were used as received without any further purification, unless otherwise described. Methacrylic acid (MAA, 99\%), 2,2'-azobisisobutyronitrile (AIBN, 98\%), anhydrous ethanol, anhydrous acetone and anhydrous diethyl ether were purchased from National Medicines Corporation Ltd. of China (Beijing, China). Pentaerythritol tetrakis 3-mercaptopropionate (PTMP, 99\%) was obtained from Aldrich (St. Louis, MO, USA). Ferric Chloride $\left(\mathrm{FeCl}_{3} \cdot 6 \mathrm{H}_{2} \mathrm{O}\right.$, $99 \%)$, ferrous sulfate $\left(\mathrm{FeSO}_{4} \cdot 7 \mathrm{H}_{2} \mathrm{O}, 99 \%\right)$, hydrochloric acid $(\mathrm{HCl}, 38 \%)$ and ammonium hydroxide $\left(\mathrm{NH}_{4} \mathrm{OH}, 28 \%\right)$ were obtained from Sinopharm Chemical Reagent Co. Ltd. (Shanghai, China) $N$-(3-dimethylamino-propyl)- $N^{\prime}$-ethylcarbodiimide hydrochloride (EDC) and $N$-hydroxysuccinimde (NHS) were purchased from Aladdin Reagent Co., Ltd. (Shanghai, China). Fetal bovine serum (FBS) and Dulbecco's modified eagle's medium (DMEM) were obtained from Gibco (Basel, Switzerland). The 3-(4,5-Dime-ltetrazolium bromide) (MTT) cell proliferation assay kit was received from Amresco (Solon, OH, USA). Milli-Q water was used in the preparation and subsequent application experiments.

\subsection{Synthesis and Characterization of Polymer Ligand PTMP-PMAA}

PTMP-PMAA was synthesized by free radical polymerization of monomer methacrylic acid (MAA) using pentaerythritol tetrakis 3-mercaptopropionate (PTMP) as chain transfer agent as described in previous reports [28-30]. The molar ratio of monomer to chain transfer agent helped to control the molecular weight of the polymer. Scheme 1 represents the polymer synthesis process.
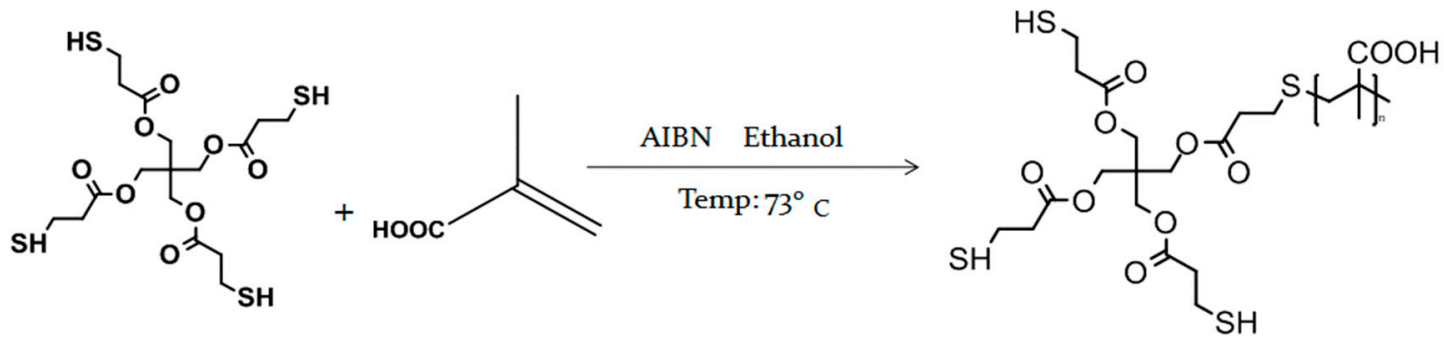

Scheme 1. Synthesis of polymer ligand pentaerythritol tetrakis 3-mercaptopropionate-polymethacrylic acid (PTMP-PMAA).

In a typical preparation for PTMP-PMAA, methacrylic acid (MAA, $5 \mathrm{~g}, 58 \mathrm{mmol}$ ), pentaerythritol tetrakis 3-mercaptopropionate (PTMP, $0.56 \mathrm{~g}, 1.16 \mathrm{mmol}, 2 \%$ of monomer) and 2,2'-azobisisobutyronitrile (AIBN, $0.095 \mathrm{~g}, 0.58 \mathrm{mmol}, 1 \%$ of monomer) were added to EtOH $(25 \mathrm{~mL})$ in a three-necked round-bottomed flask, equipped with a reflux condenser and mechanical stirrer. The temperature of the reaction mixture was maintained at $75{ }^{\circ} \mathrm{C}$ for $5 \mathrm{~h}$ under Nitrogen with vigorous stirring. At the end of this period, the reaction mixture was left to cool down to room temperature and then the products were isolated by precipitation into cold diethyl ether. The polymer was collected by filtration on a Buchner funnel, and the solvent and monomer residues were removed 
by evaporation to constant mass using a vacuum oven set at $45^{\circ} \mathrm{C}$. A fraction of low molar mass polymer, un-reacted monomer and some oligomers remaining after reaction are removed during the precipitation step. The yield obtained was $80 \%$ for this reaction. ${ }^{1} \mathrm{H}-\mathrm{NMR}$ spectra were recorded on a $400 \mathrm{MHz}$ Bruker AV400 spectrometer (Billerica, MA, USA) using $d_{6}$-DMSO as a solvent in a $5 \mathrm{~mm}$ quartz NMR tube at room temperature using the $\delta$ scale and were consistent to the previous reports $[28,29]$. The molecular weights of polymer were determined by gel permeation chromatography (GPC) on Agilent 1100 instrument (Santa Clara, CA, USA) using THF as mobile phase after its methylation with TMS-diazomethane.

\subsection{Synthesis of MIONs}

MIONs synthesis process was adopted from our previous work with few modifications $[20,25]$. Typical procedure involves the co-precipitation of aqueous iron precursor solution with ammonia in the presence of polymer ligand at high temperature (Scheme 2). Brief experimental details of the MIONs synthesis using polymer ligand PTMP-PMAA are provided in the following paragraph.
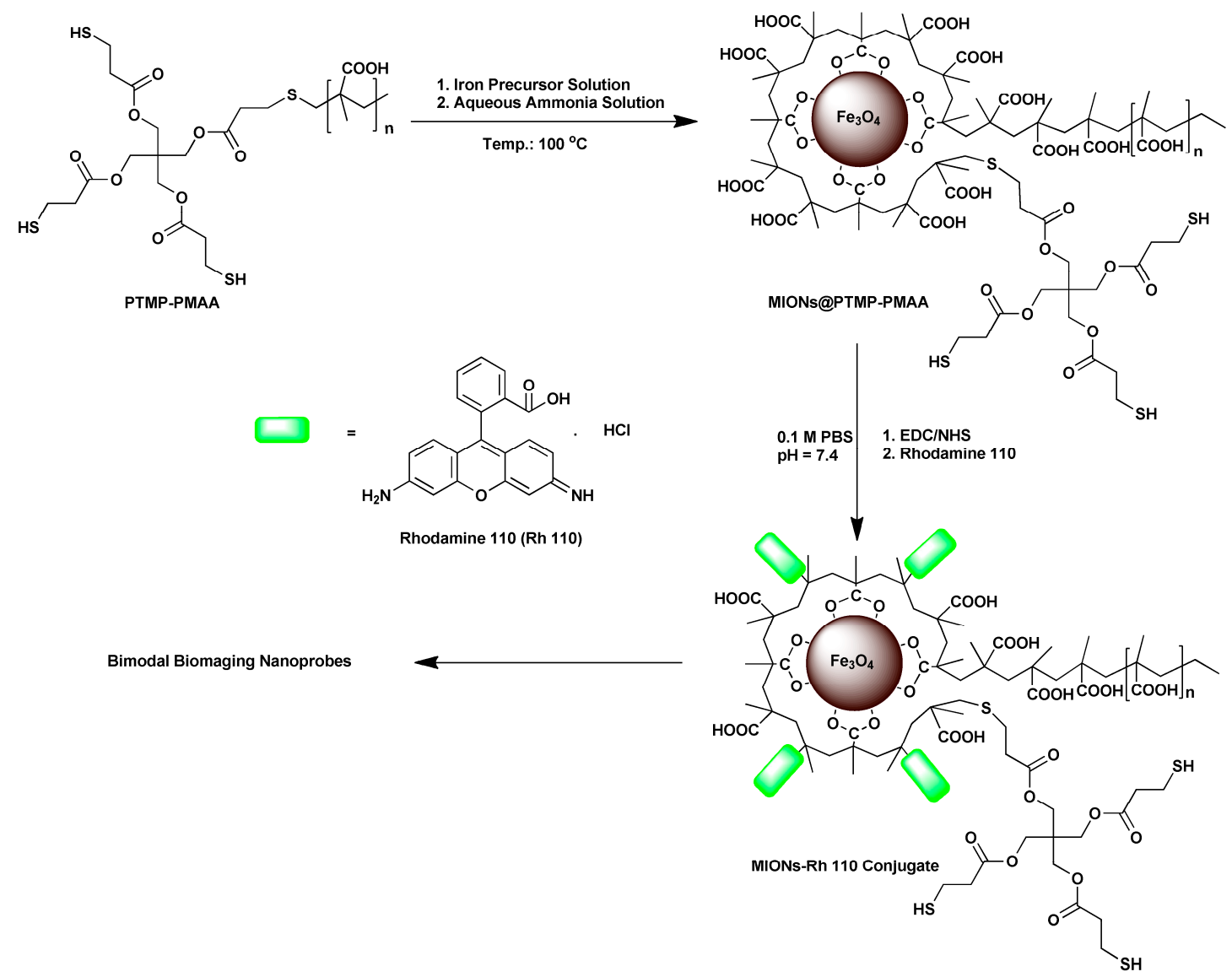

Scheme 2. Graphical representation of the synthesis of PTMP-PMAA functionalized MIONs and their subsequent functionalization with Rhodamine 110.

Briefly, in a $500 \mathrm{~mL}$ four-necked round-bottom flask equipped with reflux condenser, thermometer and nitrogen supply, $300 \mathrm{~mL}$ of Milli-Q water was added. The water was purged with nitrogen gas to remove oxygen and was heated to reflux in oil bath with magnetic stirring. When temperature reached $80^{\circ} \mathrm{C}$, the polymer ligand (PTMP-PMAA) was introduced to flask to make the aqueous solution of polymer ligand $(0.072 \mathrm{mM}, \mathrm{pH}=4)$. When the temperature reached at $100{ }^{\circ} \mathrm{C}$, the iron precursor solution comprising of $\mathrm{FeCl}_{3} \cdot 6 \mathrm{H}_{2} \mathrm{O}(3.24 \mathrm{mmol})$ and $\mathrm{FeSO}_{4} \cdot 6 \mathrm{H}_{2} \mathrm{O}(1.62 \mathrm{mmol})$ in $6 \mathrm{~mL}$ of concentration $\mathrm{HCl}$ was added and the $90 \mathrm{~mL}$ of concentration $\mathrm{NH}_{4} \mathrm{OH}$ was added within $5 \mathrm{~s}$. Upon addition of iron 
precursor solution, the color of the reaction mixture became yellow and upon addition of ammonia solution the color of the reaction mixture turned to dark black suddenly indicating the formation of iron oxide NPs. The temperature of the reaction mixture dropped to $85^{\circ} \mathrm{C}$ upon the addition of iron precursor and ammonia addition and it took $\sim 15 \mathrm{~min}$ to raise the temperature to $100{ }^{\circ} \mathrm{C}$ again and then the reaction was allowed to continue for $2 \mathrm{~h}$ at this temperature with vigorous magnetic stirring and constant nitrogen bubbling. After $2 \mathrm{~h}$ the heating was stopped and reaction mixture allowed cooling down to room temperature under nitrogen and then solvent was removed by rotary evaporator and solution concentrated to $60 \mathrm{~mL}$ and dialyzed against Milli-Q water for $72 \mathrm{~h}$ using dialysis membrane with molecular weight cut-off value 14,000 kDa. The dried NPs powder was obtained by evaporation of dialyzed NPs black suspension using rotary evaporator, washing with acetone and then drying in vacuum oven to a constant weight. The yield obtained was $\sim 90 \%$. Different samples of MIONs were prepared in the same way but with different concentrations of polymer ligand PTMP-PMAA, in order to obtain MIONs with optimum size and magnetic properties.

\subsection{Characterization of MIONs}

The particle size of the MIONs was determined by a high performance particle sizer (Brookehaven Nano DLS 90Plus/BI-MAS, Holtsville, NY, USA) with multi angle particle sizing option (from Brookhaven Instruments Co., Holtsville, NY, USA) with an effective detection capability of 0.6 to $6000 \mathrm{~nm}$. The zeta potential values were determined by a Brookhaven ZetaPlus (Brookhaven Instruments Co., Holtsville, NY, USA) at $25^{\circ} \mathrm{C}$ using the folded capillary cells. Data were obtained using a monomodal acquisition and was fit according to the Smoluchowski theory. After filtration with aqueous membrane $(\Phi=13 \mathrm{~mm}, 0.22 \mu \mathrm{m})$, the samples were analyzed using ultrapure water as solvent $(\mathrm{pH}=7)$. These measurements were run at least three times with independent particle batches. Transmission electron microscopy images were recorded on a JEOL-2100 electron microscope (Akishima, Tokyo, Japan) operating at an acceleration voltage of $200 \mathrm{kV}$. TEM samples were prepared by the slow evaporation of a drop of dilute aqueous solution of MIONs onto carbon-coated copper grids (400 mesh). Images were recorded with a Gatan 794 CCD camera (Pleasanton, CA, USA). Size distribution graphs were prepared by analyzing about 200 individual NPs on each TEM image by ImageJ software. X-ray diffraction (XRD) was recorded on X'Pert PRO XRD spectrometer (PANalytical B.V., Kassel, Holand) using $\mathrm{Cu} \mathrm{K} \alpha(\lambda=1.54056 \AA)$ radiation in the $2 \theta$ range of $10^{\circ}-80^{\circ}$.

The UV-vis spectra were recorded using Lambda 35 UV-vis spectrophotometer (Perkin-Elmer, Waltham, MA, USA). The infrared spectra were recorded by a Fourier transform infrared (FTIR) spectrometer (Bruker Vertex 70, Billerica, MA, USA) equipped with an attenuated total reflection (ATR) accessory. Raman spectra were measured on a HR JOBIN YVON spectrometer using a laser of 632 $\mathrm{nm}$ and a $25 \%$ filter. Thermogravimetric analysis (TGA) measurements were made using TGA Q500 (TA Instruments, New Castle, DE, USA) at a heating rate of $10^{\circ} \mathrm{C} \cdot \mathrm{min}^{-1}$ from room temperature to $900^{\circ} \mathrm{C}$ in oxygen free atmosphere. The saturation magnetizations (Ms) of the MIONs were measured at $26.8^{\circ} \mathrm{C}$ on a Lakeshore 7400 Series vibrating sample magnetometer (Lake Shore Cryotonics, Westerville, $\mathrm{OH}, \mathrm{USA}$ ). All the magnetization data were normalized to the same weight.

\subsection{Cytotoxicity Analysis of MIONs}

Toxicity analysis of the MIONs was carried out by MTT-assay using HepG2 cells. HepG2 cells were cultured in Dulbecco's Modified Eagle Medium (DMEM) supplemented with $10 \%$ fetal bovine serum (FBS), $100 \mu \mathrm{g} \cdot \mathrm{mL}^{-1}$ streptomycin and $100 \mathrm{U} \cdot \mathrm{mL}^{-1}$ penicillin, in a humidified incubator at $37^{\circ} \mathrm{C}$ with a $5 \% \mathrm{CO}_{2}$ atmosphere. Cell viability was determined by MTT-assay. HepG2 cells were seeded into a 96-well plate with a cell density of $1 \times 10^{4}$ cells per well and suspended in DMEM supplemented with $10 \% \mathrm{FBS}$ and incubated for $24 \mathrm{~h}$ at $37{ }^{\circ} \mathrm{C}$ in a $5 \% \mathrm{CO}_{2}$ atmosphere. After that, the cell culture medium was replaced with fresh medium containing different concentrations of $(25,50,100,200$, 500 and 1,000 $\mu \mathrm{g} \cdot \mathrm{mL}^{-1}$ ) MIONs, PTMP-PMAA and MIONs@PTMP-PMAA in triplicate. A control experiment with only cell culture medium without any NPs or polymer was also carried out in each 
case. Plates were placed at $37^{\circ} \mathrm{C}$ in a humidified $5 \% \mathrm{CO}_{2}$ incubator and MTT-assay was performed after 24,48 and $72 \mathrm{~h}$.

For MTT-assay, briefly cell culture media were aspirated and $20 \mu \mathrm{L}$ MTT $\left(5 \mathrm{mg} \cdot \mathrm{mL}^{-1}\right)$ [3-(4,5-dimethylthiazol-2-yl)2,5-diphenyltetrazolium bromide] in FBS free DMEM was added to each well and incubated for another night at $37^{\circ} \mathrm{C}$ in a humidified $5 \% \mathrm{CO}_{2}$ incubator. After incubation, MTT solution was removed and $150 \mu \mathrm{L}$ DMSO was added in each vial to dissolve newly formed formazan crystals. The plates were placed on a swing bed for $10 \mathrm{~min}$ and then absorbance was recorded at $490 \mathrm{~nm}$ using a micro plate reader (Thermo Electron Corporation, Waltham, MA, USA). The absorbance was recorded in triplicate in each case with subtraction for plate absorbance at $650 \mathrm{~nm}$ and percentage cell viability was calculated as the ratio of mean absorbance of triplicate readings with respect to mean absorbance of control wells:

$$
\text { Cell viability }=\left(I_{\text {sample }} / I_{\text {control }}\right) \times 100
$$

\subsection{Conjugation of MIONs@PTMP-PMAA with Rhodamine 110}

MIONs@PTMP-PMAA, EDC/NHS, and Rh 110 were separately dissolved in phosphate buffered saline (PBS) solution $(1 \mathrm{~mL}, \mathrm{pH}=6.8)$. The concentration of $\mathrm{Rh} 110$ was 0.1 and $1.0 \mathrm{mg} \cdot \mathrm{mL}^{-1}$ for the other reagents. EDC $(1.45 \mathrm{~mL})$ and NHS $(400 \mu \mathrm{L})$ were sequentially added to $\mathrm{Fe}_{3} \mathrm{O}_{4}$ solution $(10 \mathrm{~mL})$ and then $\mathrm{Rh} 110$ solution $(2.8 \mathrm{~mL})$ was added and the mixture was stirred for $24 \mathrm{~h}$ at room temperature. The resulting conjugate (MIONs-R 110) was purified by extensive dialysis against distilled water for three nights. As a control, a dye solution with same molar concentration was also dialyzed for three nights.

\section{Results and Discussion}

\subsection{Synthesis and Characterization of PTMP-PMAA}

Multi-functional water-soluble polymer ligand PTMP-PMAA was synthesized using pentaerythritol tetrakis 3-mercaptopropionate as chain transfer agent by free radical polymerization of monomer methacrylic acid (MAA) as described previously (Scheme 1). GPC elution curve of polymer is shown in Figure 1 and its molecular weights are given in Table 1.

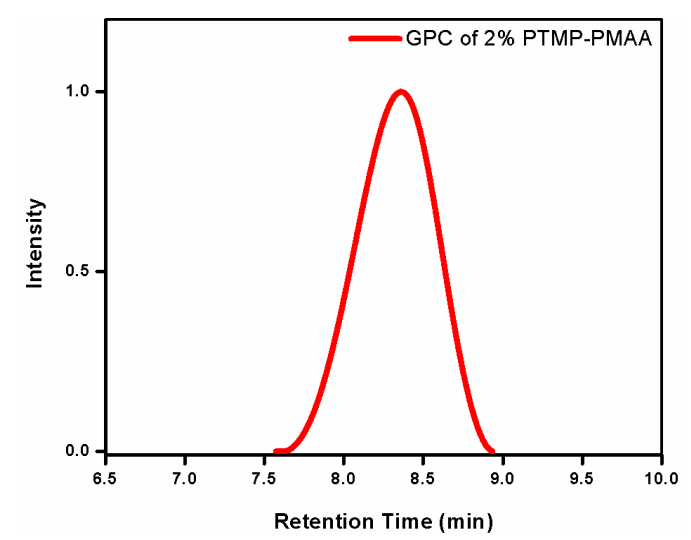

Figure 1. GPC (gel permeation chromatography) curve of the PTMP-PMAA obtained with $2 \%$ pentaerythritol tetrakis 3-mercaptopropionate (PTMP).

Table 1. Molecular weight of $\%$ PTMP-PMAA.

\begin{tabular}{cccccc}
\hline \multirow{2}{*}{ Sample } & \multirow{2}{*}{$\begin{array}{c}\text { MAA/PTMP } \\
(\mathbf{m o l} / \mathbf{m o l})\end{array}$} & \multicolumn{3}{c}{ Molecular weights (g/mol) } & \multirow{2}{*}{ Yield (\%) } \\
\cline { 3 - 5 } & & $\mathbf{M n}$ & $\boldsymbol{M w}$ & PDI & \\
\hline 2\% PTMP-PMAA & $100 / 2$ & 5,850 & 7,420 & 1.2 & 82 \\
\hline
\end{tabular}


${ }^{1} \mathrm{H}-\mathrm{NMR}$ spectra were recorded on a $400 \mathrm{MHz}$ Bruker AV400 spectrometer using $d_{6}$-DMSO as a solvent in a $5 \mathrm{~mm}$ quartz NMR tube at room temperature using the $\delta$ scale. The ${ }^{1} \mathrm{H}-\mathrm{NMR}$ spectra were consistent to the previous works $[28,29]$. The molecular weights of the polymer ligands were calculated based on the ratio of monomer units attached to the terminal group in the ${ }^{1} \mathrm{H}-\mathrm{NMR}$ spectra and compared with molecular weights determined by GPC (data not given). Chemical structure of the polymer was confirmed by ${ }^{1} \mathrm{H}-\mathrm{NMR}$ spectroscopy and data as given were consistent with our previous work $[28,29]$.

PTMP-PMAA ( $d_{6}$-DMSO) $\delta$ (ppm): $\delta 0.81 \sim 1.14 ; \delta 1.42 \sim 1.54 ; \delta 1.61 \sim 1.28 ; \delta 2.24 \sim 2.40 ; \delta 2.51 \sim 2.84 ;$ 83.17 3.50; 83.91 4.40.

The as synthesized polymer was soluble in $\mathrm{EtOH}, \mathrm{MeOH}, \mathrm{H}_{2} \mathrm{O}$ and DMSO. In order to determine the molecular weight of polymer ligand by GPC it was transferred to the THF by converting it into methyl ester using TMS-Diazomethane reagent according to the previous work [30,31]. GPC was performed with an Agilent 1100 instrument using refractive index detector (RID) and THF was used as eluent at a flow rate of $1.0 \mathrm{~mL} / \mathrm{min}$ at $23^{\circ} \mathrm{C}$. The calculated molecular weights were based on a calibration curve for polystyrene standards of narrow polydispersity (Polymer Laboratories). As expected, the molecular weight of the polymer was decreased with the increase in concentration of chain transfer agent.

\subsection{Synthesis and Characterization of Polymer Stabilized MIONs}

MIONs were synthesized by co-precipitation of aqueous iron precursor solution containing $\mathrm{Fe}^{3+}$ and $\mathrm{Fe}^{2+}$ (molar ratio 2:1) by ammonia in the presence of PTMP-PMAA. The size, shape and magnetic properties of the MIONs were controlled by using different molecular weight and concentration of polymer ligand, during their preparation. This polymer ligand has already been proven to be a good capping ligand for the synthesis of cobalt and gold NPs by some of us [21,28,29].

It is known that rapid injection of precursors results in super saturation of chemical species in the reaction mixture which leads to an initial burst of nucleation at once followed by the growth of nuclei leading to the formation of monodisperse inorganic NPs, whereas slow drop-wise addition of precursors results in continuous nucleation and growth process in parallel resulting in broad size distribution of NPs [32]. However, in the case of magnetite NPs, the process is more complicated and it is difficult to control the nucleation and growth processes because iron precursors are initially hydrolyzed in alkaline conditions and then are condensed into iron oxide. Therefore, we dissolved iron precursors in concentrated $\mathrm{HCl}(38 \%)$ in order to avoid their hydrolysis and condensation prior to the addition of precipitating agents. Both iron precursor's solution and ammonia were rapidly added into the boiling aqueous solution of polymer under nitrogen atmosphere with vigorous stirring in order to keep nucleation and growth processes separate. Rapid injection of precursor is widely used for uniform inorganic NPs synthesis in organic phase by thermal decomposition method [32,33]. The preparation of ultra-small and uniform magnetic NPs with high crystallinity and magnetization through usual co-precipitation method at room temperature is a tedious job, therefore we have carried out co-precipitation process at high temperature $\left(100{ }^{\circ} \mathrm{C}\right)$ [20], which increases the reaction rate due to increased diffusion of active species resulting in the formation of NPs with a narrow size distribution [20].

Polymer ligand (PTMP-PMAA) molecules cap the newly formed NPs through interaction between carboxylic acids groups and the iron atoms on NPs surface and thus restrict their further growth by compensating the surface energy due to electrostatic and steric stabilization which yields uniform as well as highly stable NPs. We have already proved this hypothesis by taking samples at different time intervals showing little further growth of NPs with the passage of time [20]. It was also noticed that the use of different ferrous precursor $\left(\mathrm{FeCl}_{2} \cdot 4 \mathrm{H}_{2} \mathrm{O}\right.$ instead of $\left.\mathrm{FeSO}_{4} \cdot 7 \mathrm{H}_{2} \mathrm{O}\right)$ had no effect on shape, size and size distribution of the MIONs. Moreover, in comparison to the thermal decomposition method, this method involves no use of expensive and toxic organic precursors or solvents but reduces the reaction time and temperature; therefore, it is more economical and eco-friendly. 


\subsubsection{Effect of Polymer Concentration on Size of MIONs}

As described earlier the purpose of this research work was to improve the magnetic properties of our previous reported MIONs prepared with PTMP-PMAA [20]. In that report, MIONs prepared with $0.768 \mathrm{mM}$ concentration of PTMP-PMAA had hydrodynamic diameter $<10 \mathrm{~nm}$ determined by DLS and core size of $4.5 \pm 0.4 \mathrm{~nm}$ as determined by TEM. Those MIONs were highly water soluble but their small size and lower magnetization $\left(45 \mathrm{emu} \cdot \mathrm{g}^{-1}\right)$ restricted their applications such as magnetic separation. Therefore, herein, we gradually decreased the concentration of PTMP-PMAA from $0.768 \mathrm{mM}$ all the way down to zero during preparation of MIONs and optimized the polymer concentration to obtain MIONs with higher saturation magnetization without compromising their size, dispersibility and stability.

Figure 2a shows the DLS curves of the MIONs prepared with different PTMP-PMAA concentrations and it was noticed that the hydrodynamic diameters of the MIONs prepared with polymer concentrations as low as $0.072 \mathrm{mM}$ did not result in the increase in the size of the MIONs. However, when the polymer concentration was decreased beyond $0.072 \mathrm{mM}$, the size of the MIONs noticeably increased. Figure $2 \mathrm{~b}$ represent the DLS curves of the MIONs prepared with 0.072 and $0.768 \mathrm{mM}$ PTMP-PMAA representing their hydrodynamic diameters to be 9 and $10 \mathrm{~nm}$ respectively, which strongly indicates that the a ten times decrease in polymer concentration did not much affect the size of the MIONs. Therefore, $0.072 \mathrm{mM}$ was considered to be the lowest effective polymer ligand concentration and thus the MIONs prepared with $0.072 \mathrm{mM}$ PTMP-PMAA were selected for further characterization through TEM analysis and the results were compared with those of 0.768 mM PTMP-PMAA.

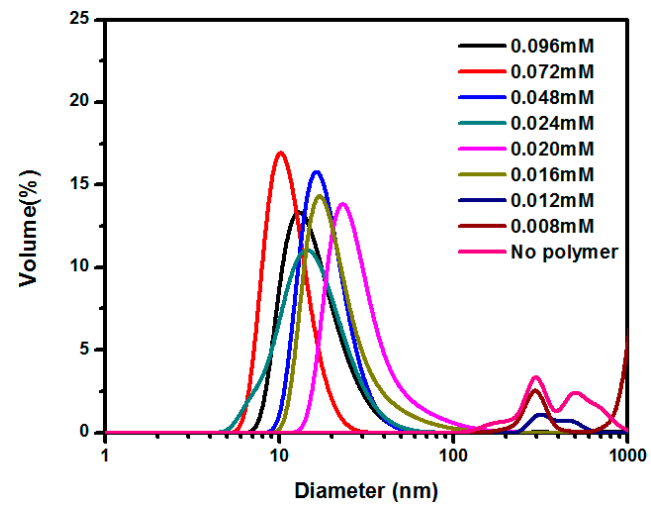

(a)

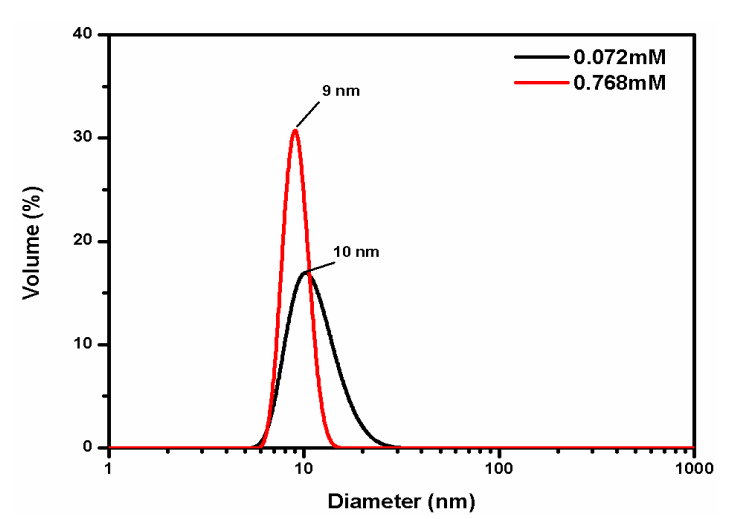

(b)

Figure 2. (a) Dynamic light scattering (DLS) curves of the MIONs prepared with different concentrations of PTMP-PMAA all lower than $0.768 \mathrm{mM}$; and (b) DLS curves of the MIONs prepared with 0.768 and $0.072 \mathrm{mM}$ concentration of PTMP-PMAA.

In order to determine the actual iron oxide core size of these MIONs, we performed the TEM analysis of the MIONs prepared with 0.072 and $0.768 \mathrm{mM}$ concentrations of PTMP-PMAA. It was interesting to note that the actual size of the MIONs was almost half of their hydrodynamic diameters suggesting the presence of extended polymer shell around these nanoparticles when dispersed in aqueous solutions. The TEM size of the MIONs prepared with $0.768 \mathrm{mM}$ PTMP-PMAA was found to be $4.6 \mathrm{~nm}$ with a narrow size distribution of $0.4 \mathrm{~nm}$ (Figure 3a) and these results were in complete agreement with the previous report [20]. However, the TEM analysis of the MIONs prepared with $0.072 \mathrm{mM}$ PTMP-PMAA revealed that their size was $4.8 \mathrm{~nm}$ with a size distribution of $0.6 \mathrm{~nm}$ (Figure $3 \mathrm{~b}$ ), which is very much comparable to the MIONs prepared with $0.768 \mathrm{mM}$ PTMP-PMAA. The slight increase in size and size distribution of these MIONs in comparison to the ten times decrease in the polymer concentration can be considered as insignificant and legitimate. 


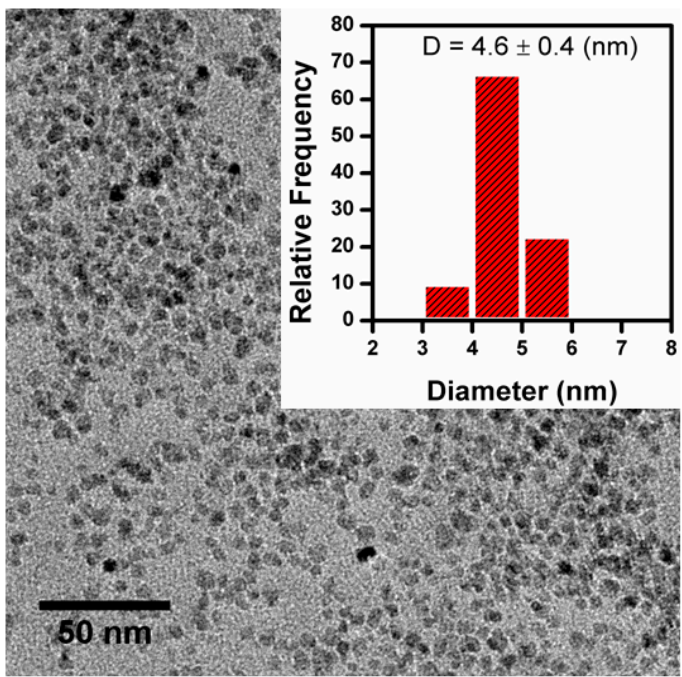

(a)

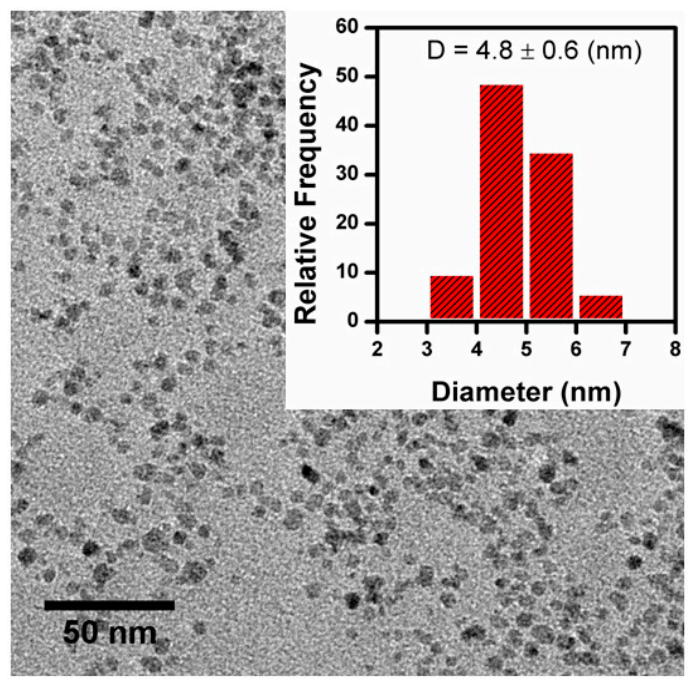

(b)

Figure 3. TEM image and histogram (insert) of MIONs prepared with: (a) 0.768 mM PTMP-PMAA; and (b) 0.072 mM PTMP-PMAA.

Conversely, the MIONs obtained without PTMP-PMAA had TEM diameter more than $10 \mathrm{~nm}$ with very large size distribution range $2.9 \mathrm{~nm}$ (Figure 4) and were not very well dispersed in aqueous solution as they tended agglomerate and settle down under force of gravity within a few minutes, perhaps due to their large size, high surface energy, etc. These results revealed that the concentration of the PTMP-PMAA (carbonyl groups to iron precursor ratio) played an important role, and is a critical factor in determining size and size-distribution of the MIONs. It should be noted that the DLS diameter of the bare MIONs was observed to be more than $70 \mathrm{~nm}$ because of their high agglomeration, which was further confirmed by TEM (Figure 4).

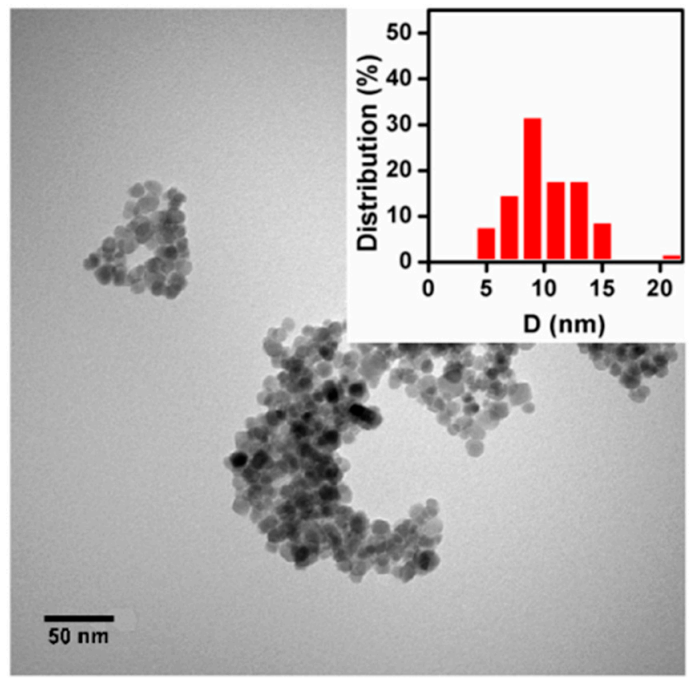

Figure 4. TEM image of Bare MIONs with histogram (inset).

In conclusion, it was found that the MIONs were successfully synthesized with small size $(4.8 \pm 0.6 \mathrm{~nm})$ using $0.072 \mathrm{mM}$ PTMP-PMAA which is almost ten times lower concentration as compared to $0.768 \mathrm{mM}$ used previously without significant increase in the size and size distribution of the MIONs. Furthermore, it would also improve the magnetic properties of the MIONs by lowering the polymer contents percentage. 


\subsubsection{Characterization of MIONs}

Further characterization of the MIONs was performed by taking FTIR, TGA, XRD and VSM analysis of the MIONs prepared with 0.072 and $0.768 \mathrm{mM}$ PTMP-PMAA, and results were compared.

In order to know the surface chemistry of MIONs, they were characterized by Fourier transform infrared spectroscopy (Figure 5). In FTIR spectrum of polymer ligand, a strong absorption peak at $1704 \mathrm{~cm}^{-1}$ corresponds to the asymmetric stretching of carbonyl (-CO-) group of polymer ligand, which is extensively reduced in spectrum of MIONs with polymer indicating the attachment to the surface of iron oxide NPs [34]. Additionally, a broad peak at $3124 \mathrm{~cm}^{-1}$ is attributed to the $\mathrm{O}-\mathrm{H}$ stretching of carboxylic acid groups and is observed in FTIR spectra of PTMP-PMAA and MIONs@PTMP-PMAA [28,35]. Furthermore, the asymmetric and symmetric stretching bands of carboxylate (-COO-) present in pure polymer ligand spectrum are shifted from 1485, 1390 to 1556 and $1402 \mathrm{~cm}^{-1}$, respectively, in the spectrum of MIONs protected with polymer ligand, which confirms the presence of polymer ligand on NPs surface. Finally, the characteristic peaks appearing at 592 and $448 \mathrm{~cm}^{-1}$ are assigned to the torsion vibration and stretching mode of $\mathrm{Fe}-\mathrm{O}$ bond of magnetite present only in FTIR spectra of MIONs with PTMP-PMAA $[35,36]$.

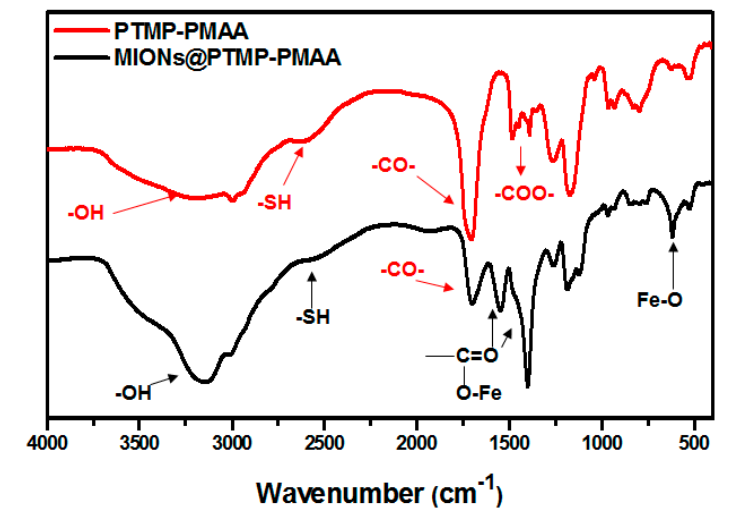

Figure 5. Fourier transform infrared spectroscopy (FTIR) spectra of PTMP-PMAA and MIONs@PTMP-PMAA.

The crystalline structure of the MIONs was determined by XRD measurement as shown in Figure 6 . The peaks present at $2 \theta=30.1^{\circ}, 35.5^{\circ}, 43.3^{\circ}, 53.4^{\circ}, 57.3^{\circ}$ and $62.7^{\circ}$ corresponding to $(220)$, (311), (400), (422), (511) and (440) reflections of magnetite, respectively, can be clearly seen, indicating the presence of crystalline spinel structured magnetite $\left(\mathrm{Fe}_{3} \mathrm{O}_{4}\right)$ phase of iron oxide [37]. However, the presence of $\mathrm{Fe}_{2} \mathrm{O}_{3}$ is not completely ruled out as the phase of the iron oxide in magnetic NPs cannot be confirmed from simple FTIR and XRD as they are less sensitive to them [20].

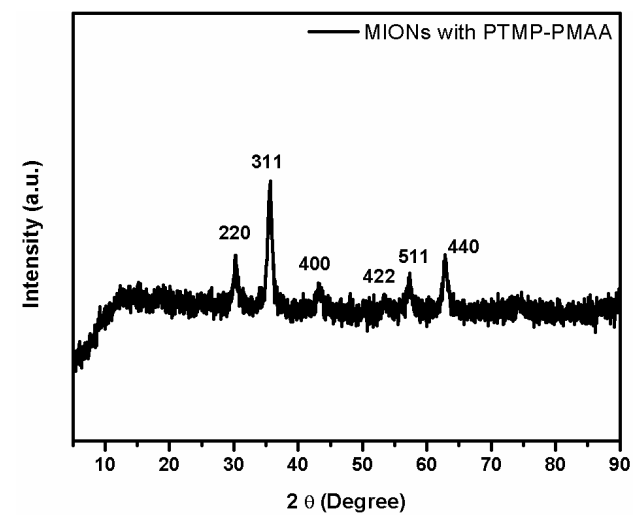

Figure 6. X-ray powder diffraction (XRD) spectrum of MIONs prepared with PTMP-PMAA. 
To further investigate the thermal stability of the MIONs, and to deduce the iron oxide and polymer contents of the NPs, thermal gravimetric analysis was performed for PTMP-PMAA, MIONs prepared with 0.072 and $0.768 \mathrm{mM}$ PTMP-PMAA as well as for bare MIONs prepare without the use of any polymer ligand (Figure 7). It can be seen that neat polymer ligand (PTMP-PMAA) was almost completely decomposed at $480{ }^{\circ} \mathrm{C}$ with almost no residue left. The magnetic iron oxide and polymer contents of the MIONs prepared with 0.768 mM PTMP-PMAA were approximately $33 \%$ and $67 \%$, respectively. However, the iron oxide and polymer contents of the MIONs prepared with $0.072 \mathrm{mM}$ PTMP-PMAA were $42 \%$ and $58 \%$, respectively, as expected. Therefore, from TGA analysis, it was concluded that the magnetic iron oxide wt $\%$ in the MIONs with PTMP-PMAA $(0.072 \mathrm{mM})$ was higher than those prepared with $0.768 \mathrm{mM}$ PTMP-PMAA which contributed towards the higher saturation magnetization of the MIONs in this case.

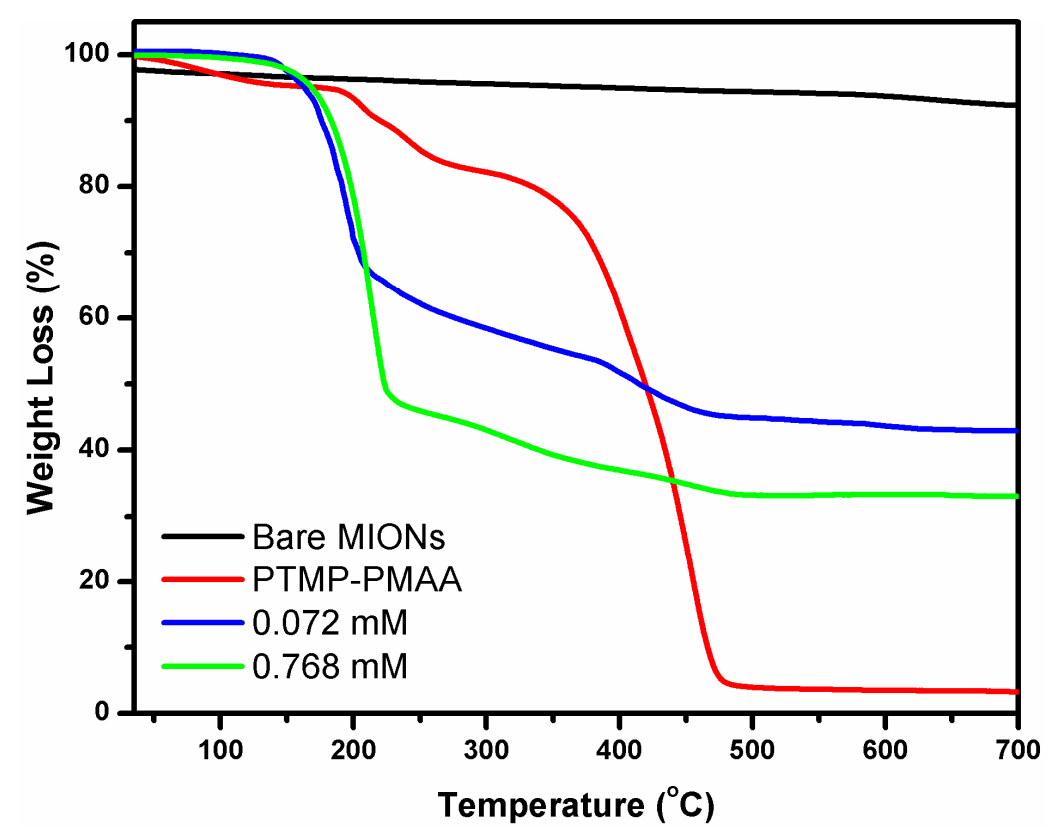

Figure 7. Thermogravimetric analysis (TGA) curves of PTMP-PMAA, MIONs with PTMP-PMAA (0.072 $\mathrm{mM}$ and $0.768 \mathrm{mM})$ and bare MIONs.

Saturation magnetization of MIONs prepared with 0.072 and $0.768 \mathrm{mM}$ PTMP-PMAA ligands was determined by Vibrating Sample Magnetometer (VSM) at room temperature. In order to calculate the exact magnetization of these MIONs, the effect of magnetically dead polymer layer was excluded and then compared with magnetization of bare MIONs without polymer (Figure 8). The corrected saturation magnetization of the MIONs prepared with $0.768 \mathrm{mM}$ PTMP-PMAA, having TEM diameter about $4.6 \mathrm{~nm}$, was found to be $45 \mathrm{emu} \cdot \mathrm{g}^{-1}$, which is comparable to the literature for MIONs of comparable dimensions [20]. The low saturation magnetization of $4.6 \mathrm{~nm}$ MIONs compared to the bare MIONs is due to their ultra-small size as saturation magnetization of magnetic NPs is directly related to their size [38]. However, it was interesting to note that the saturation magnetization of our MIONs prepared with $0.072 \mathrm{mM}$ PTMP-PMAA with TEM diameter $4.8 \mathrm{~nm}$ was found to be $58 \mathrm{emu} \cdot \mathrm{g}^{-1}$, which is much higher than that of MIONs prepared with $0.768 \mathrm{mM}$. We attribute this increase in the saturation magnetization of the MIONs to the decrease in magnetically dead polymer contents of the MIONs as mentioned earlier. 


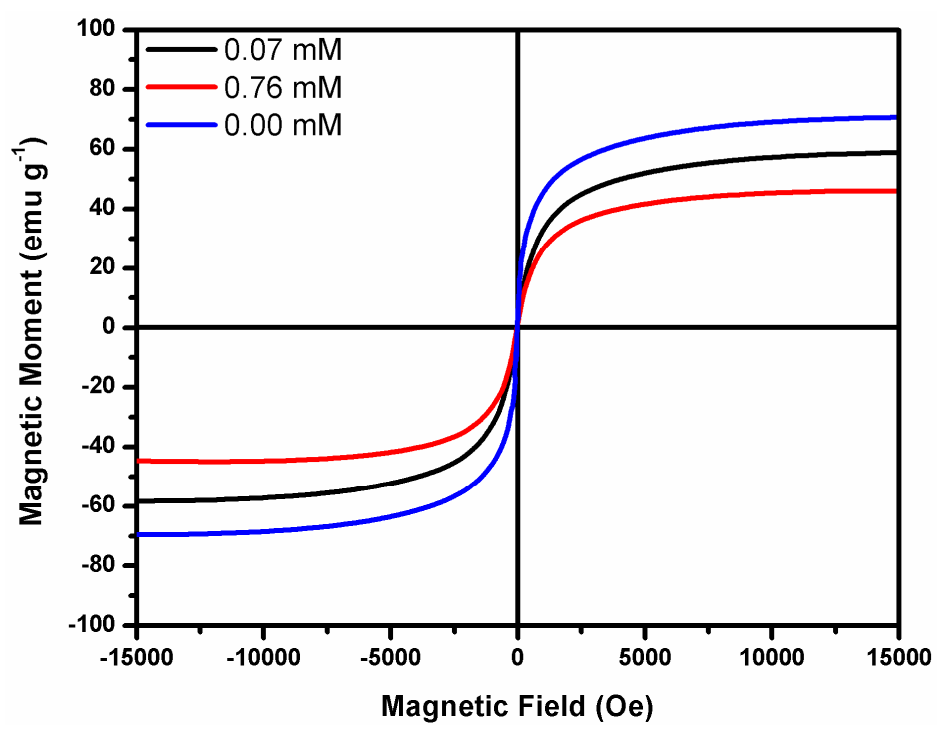

Figure 8. Magnetization curves of MIONs prepared with $0.072,0.768$ and $0.00 \mathrm{mM}$ concentration of PTMP-PMAA.

However, the magnetization of the MIONs was still much lower than that of bare MIONs prepared without polymer ligand (Figure 8). Therefore, it can be concluded that water-soluble MIONs with comparable size were prepared with ten times less polymer concentration as compared to previous report [20], which resulted in a great increase in the magnetization of the MIONs.

It is interesting to note that the increase in the saturation magnetization of the MIONs made them able to be collected with the help of an external magnet from their aqueous solution without compromising their stability and dispersibility, as shown in the Figure 9. We believe that this would increase the scope of applications of these MIONs such as the magnetic separations of biomolecules like proteins, nucleic acids and even cells.

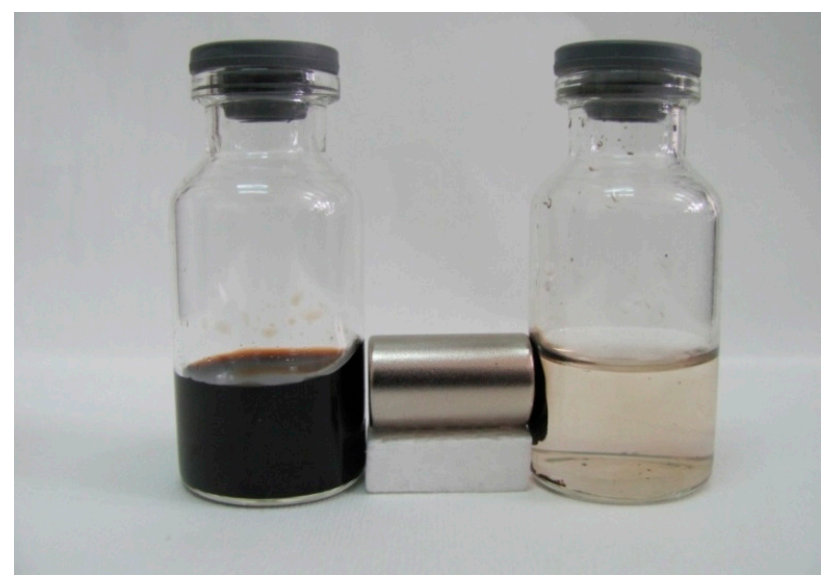

Figure 9. Aqueous dispersion of the MIONs prepared with: $0.768 \mathrm{mM}$ (left); and $0.072 \mathrm{mM}$ (right) concentrations of PTMP-PMAA under the effect of external magnetic (1.5 T) after $1 \mathrm{~h}$.

Dried MIONs prepared with PTMP-PMAA were highly stable and readily redispersible in water, $\mathrm{pH}$ and salt stability tests were performed with aqueous dispersions of MIONs prepared with PTMP-PMAA $(0.072 \mathrm{mM})$ and were found to be stable within a wide range of $\mathrm{pH}(5-10)$ and high concentrations $(1 \mathrm{M})$ of salt $(\mathrm{NaCl})$. High stability of the MIONs dispersions can be attributed to large negative zeta potential $(-49 \mathrm{mV})$ and excellent electrostatic as well as steric stabilization by polymer ligand owing to the large abundance of carboxylic acid groups [25]. 


\subsubsection{Toxicity Analysis of MIONs}

Biocompatibility of the MIONs@PTMP-PMAA was determined through their in vitro cytotoxicity analysis performed on HepG2 cells as demonstrated in experimental section. Figure 10 shows the percent cell viability of HepG2 cells, which was determined by MTT-assay, after incubation of cells with different concentrations of MIONs prepared with $0.072 \mathrm{mM}$ PTMP-PMAA for 24, 48 and $72 \mathrm{~h}$. Cell viability determined at zero concentration of MIONs or polymer ligand was taken as $100 \%$. These in vitro cytotoxicity tests clearly demonstrate that MIONs@PTMP-PMAA were completely non-toxic and cells survived well even at higher concentrations $\left(500 \mu \mathrm{g} \cdot \mathrm{mL}^{-1}\right)$ due to nontoxic nature of PTMP-PMAA [5], however, cell viability more than $100 \%$ in few cases was maybe due to cell proliferation. In opposite to that, bare MIONs have time and dose dependent high toxicity as described by us previously [25], which was attributed to the known fact that bare MIONs act as a source of ferrous ions and highly reactive hydroxyl radicals causing oxidative and free radical stress to the cells [39].

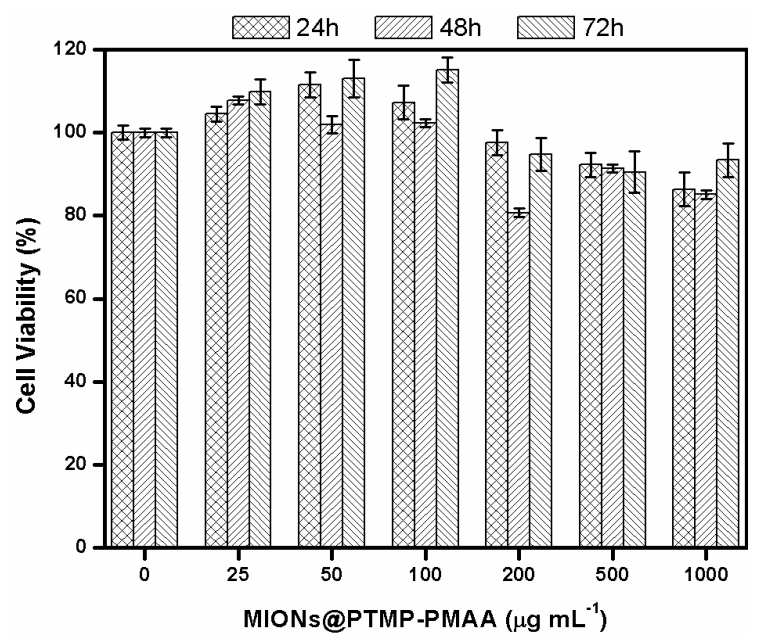

Figure 10. Cell viability of HepG2 determined by MTT-assay after 24, 48 and $72 \mathrm{~h}$ of incubation with various concentrations of MIONs@PTMP-PMAA.

\subsection{Functionalization of MIONs with Fluorescent Dye}

It was determined through characterization that MIONs@PTMP-PMAA had high water dispersibility and excellent stability in a wide range of $\mathrm{pH}$ and salt concentration and multifunctional surface chemistry, due to polymer shell around them, which make them highly suitable for bio-applications such as contrast agents for MRI, magnetic separation of biomolecules and cells, and development of NPs based drug delivery systems. Furthermore, the presence of abundant of carboxylic acid (-COOH) and thiol (-SH) groups on MIONs surface also provide great opportunities to simultaneously modify these NPs with targeting, tracing and therapeutic agents for multipurpose bio-probes. We demonstrated the functionalization of these MIONs with a fluorescent dye molecule, Rhodamine 110 (Rh 110) bearing primary amine group through EDC/NHS coupling reaction, which is the easiest and most feasible reaction of linking molecules through primary amine and carboxylic acid groups by amide bond formation.

MIONs were conjugated with Rh 110 by following the procedure described in Section 2.6. Aqueous solution of MIONs-Rh 110 conjugates, after extensive dialysis for three nights against distilled water, showed fluoresce under UV light as shown in the Figure 11. As a control, a dye solution with same molar concentration was also dialyzed for three nights. It was noted that the control experiment with equivalent concentration of $\mathrm{Rh} 110$ did not exhibit any fluorescence after dialysis, which was the indication of attachment of Rh 110 with MIONs. 


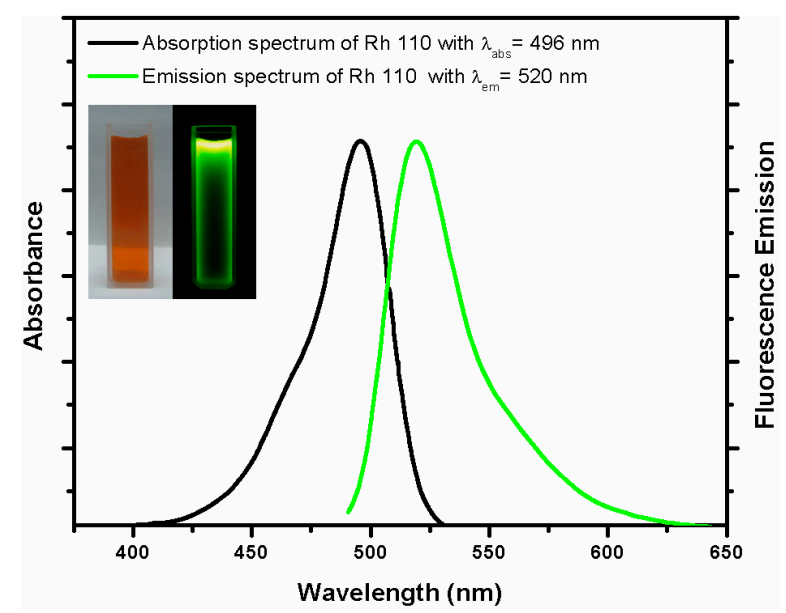

Figure 11. The UV-vis absorption (black) and fluorescent emission spectra of aqueous solution of MIONs-Rh 110 conjugate after extensive dialysis, insert shows optical image in ambient light (left), and in UV light (right).

\section{Conclusions}

Biocompatible ultra-small magnetic iron oxide $\left(\mathrm{Fe}_{3} \mathrm{O}_{4}\right)$ NPs were prepared by co-precipitation of aqueous iron precursor solution at high temperature in the presence of water-soluble thioether end-functionalized polymer ligand PTMP-PMAA. The saturation magnetization of the MIONs was further improved by optimizing the polymer ligand concentration compared to our previous work. The increase in magnetization of the MIONs made it possible to manipulate them with external magnets in their aqueous solution, which increases their scope of applications. Furthermore, it was found that these MIONs were fairly uniform in size and could be dried by solvent evaporation, which could be stored for a long time. The dried powders of MIONs were readily re-dispersible in water without any significant change in their size and size distribution. MIONs aqueous dispersion was highly stable, even at high salt concentration and also over a wide range of $\mathrm{pH}$. The concentration of polymer ligand showed significant effect on the size and magnetic properties of MIONs. MIONs were found to be highly biocompatible as determined by MTT-assay. The bio-applicability of MIONs demonstrated by successfully coupling them with fluorescent dye Rhodamine (Rh 110) through formation of amide bond linkage between carboxylic acid $(-\mathrm{COOH})$ groups of MIONs and primary amine $\left(-\mathrm{NH}_{2}\right)$ groups of dye. Similarly, rich surface chemistry of these MIONs stabilized with PTMP-PMAA can be exploited for the simultaneous conjugation of a variety of therapeutic, targeting, and labeling agents for several bio-analytical and drug delivery applications.

Acknowledgments: This work was supported by the Natural Science Foundation of Hubei Province (Q20141006), China; and Opening Funding from Ministry of Education for Key Materials and Systems for Large Scale Battery Laboratory, China.

Author Contributions: This article is a result of a valuable contribution from all the listed co-authors. Bien Tan encouraged the idea of the study and provided valuable advice; Muhammad Irfan Majeed designed and conducted the main research work; Jiaojiao Guo contributed in compiling the results and Wei Yan helped in writing this manuscript and in correspondence.

Conflicts of Interest: The authors declare no conflict of interest.

\section{References}

1. Zhang, L.; Dong, W.F.; Sun, H.B. Multifunctional superparamagnetic iron oxide nanoparticles: Design, synthesis and biomedical photonic applications. Nanoscale 2013, 5, 7664-7684. [CrossRef] [PubMed]

2. Colombo, M.; Carregal-Romero, S.; Casula, M.F.; Gutierrez, L.; Morales, M.P.; Bohm, I.B.; Heverhagen, J.T.; Prosperi, D.; Parak, W.J. Biological applications of magnetic nanoparticles. Chem. Soc. Rev. 2012, 41, 4306-4334. [CrossRef] [PubMed] 
3. Wei, W.; Zhaohui, W.; Taekyung, Y.; Changzhong, J.; Woo-Sik, K. Recent progress on magnetic iron oxide nanoparticles: Synthesis, surface functional strategies and biomedical applications. Sci. Technol. Adv. Mater. 2015, 16, 023501.

4. Yan, K.; Li, H.; Li, P.; Zhu, H.; Shen, J.; Yi, C.; Wu, S.; Yeung, K.W.K.; Xu, Z.; Xu, H.; et al. Self-assembled magnetic fluorescent polymeric micelles for magnetic resonance and optical imaging. Biomaterials 2014, 35, 344-355. [CrossRef] [PubMed]

5. Li, Z.; Yi, P.W.; Sun, Q.; Lei, H.; Zhao, H.L.; Zhu, Z.H.; Smith, S.C.; Lan, M.B.; Lu, G.Q.M. Ultrasmall water-soluble and biocompatible magnetic iron oxide nanoparticles as positive and negative dual contrast agents. Adv. Funct. Mater. 2012, 22, 2387-2393. [CrossRef]

6. Blanco-Andujar, C.; Ortega, D.; Southern, P.; Pankhurst, Q.A.; Thanh, N.T.K. High performance multi-core iron oxide nanoparticles for magnetic hyperthermia: Microwave synthesis, and the role of core-to-core interactions. Nanoscale 2015, 7, 1768-1775. [CrossRef] [PubMed]

7. Ramesh, R.; Ponnusamy, S.; Muthamizhchelvan, C. Synthesis, properties and heating characteristics of bovine serum albumin coated $\mathrm{Fe}_{3} \mathrm{O}_{4}$ magnetic fluid for magnetic fluid hyperthermia application. Sci. Adv. Mater. 2013, 5, 1250-1255. [CrossRef]

8. Lu, W.; Ling, M.; Jia, M.; Huang, P.; Li, C.; Yan, B. Facile synthesis and characterization of polyethylenimine-coated $\mathrm{Fe}_{3} \mathrm{O}_{4}$ superparamagnetic nanoparticles for cancer cell separation. Mol. Med. Rep. 2014, 9, 1080-1084. [PubMed]

9. Gao, F.; Qu, H.; Duan, Y.; Wang, J.; Song, X.; Ji, T.; Cao, L.; Nie, G.; Sun, S. Dopamine coating as a general and facile route to biofunctionalization of superparamagnetic $\mathrm{Fe}_{3} \mathrm{O}_{4}$ nanoparticles for magnetic separation of proteins. RSC Adv. 2014, 4, 6657-6663. [CrossRef]

10. Quinto, C.A.; Mohindra, P.; Tong, S.; Bao, G. Multifunctional superparamagnetic iron oxide nanoparticles for combined chemotherapy and hyperthermia cancer treatment. Nanoscale 2015, 7, 12728-12736. [CrossRef] [PubMed]

11. Oka, C.; Ushimaru, K.; Horiishi, N.; Tsuge, T.; Kitamoto, Y. Core-shell composite particles composed of biodegradable polymer particles and magnetic iron oxide nanoparticles for targeted drug delivery. J. Magn. Magn. Mater. 2015, 381, 278-284. [CrossRef]

12. Martin, M.; Salazar, P.; Villalonga, R.; Campuzano, S.; Pingarron, J.M.; Gonzalez-Mora, J.L. Preparation of core-shell $\mathrm{Fe}_{3} \mathrm{O}_{4} @$ poly(dopamine) magnetic nanoparticles for biosensor construction. J. Mater. Chem. B 2014, 2, 739-746. [CrossRef]

13. Barrow, M.; Taylor, A.; Murray, P.; Rosseinsky, M.J.; Adams, D.J. Design considerations for the synthesis of polymer coated iron oxide nanoparticles for stem cell labelling and tracking using MRI. Chem. Soc. Rev. 2015, 44, 6733-6748. [CrossRef] [PubMed]

14. Palui, G.; Aldeek, F.; Wang, W.; Mattoussi, H. Strategies for interfacing inorganic nanocrystals with biological systems based on polymer-coating. Chem. Soc. Rev. 2015, 44, 193-227. [CrossRef] [PubMed]

15. Hood, A.M.; Mari, M.; Muñoz-Espí, R. Synthetic strategies in the preparation of polymer/inorganic hybrid nanoparticles. Materials 2014, 7, 4057-4087. [CrossRef]

16. Xin, H.; Hui, Z.; Liyun, L.; Bien, T. Preparation of nanoparticles with multi-functional water-soluble polymer ligands. Prog. Chem. 2010, 22, 953-961.

17. Ling, D.; Hackett, M.J.; Hyeon, T. Surface ligands in synthesis, modification, assembly and biomedical applications of nanoparticles. Nano Today 2014, 9, 457-477. [CrossRef]

18. Wang, W.; Ji, X.; Na, H.B.; Safi, M.; Smith, A.; Palui, G.; Perez, J.M.; Mattoussi, H. Design of a multi-dopamine-modified polymer ligand optimally suited for interfacing magnetic nanoparticles with biological systems. Langmuir 2014, 30, 6197-6208. [CrossRef] [PubMed]

19. Pernia Leal, M.; Rivera-Fernandez, S.; Franco, J.M.; Pozo, D.; de la Fuente, J.M.; Garcia-Martin, M.L. Long-circulating pegylated manganese ferrite nanoparticles for mri-based molecular imaging. Nanoscale 2015, 7, 2050-2059. [CrossRef] [PubMed]

20. Li, Z.; Tan, B.; Allix, M.; Cooper, A.I.; Rosseinsky, M.J. Direct coprecipitation route to monodisperse dual-functionalized magnetic iron oxide nanocrystals without size selection. Small 2008, 4, 231-239. [CrossRef] [PubMed]

21. Lu, L.T.; Tung, L.D.; Robinson, I.; Ung, D.; Tan, B.; Long, J.; Cooper, A.I.; Fernig, D.G.; Thanh, N.T.K. Size and shape control for water-soluble magnetic cobalt nanoparticles using polymer ligands. J. Mater. Chem. 2008, 18, 2453-2458. [CrossRef] 
22. Razzaque, S.; Hussain, S.; Hussain, I.; Tan, B. Design and utility of metal/metal oxide nanoparticles mediated by thioether end-functionalized polymeric ligands. Polymers 2016, 8, 156. [CrossRef]

23. Oanh, V.T.K.; Lam, T.D.; Thu, V.T.; Lu, L.T.; Nam, P.H.; Tam, L.T.; Manh, D.H.; Phuc, N.X. A novel route for preparing highly stable $\mathrm{Fe}_{3} \mathrm{O}_{4}$ fluid with poly(acrylic acid) as phase transfer ligand. J. Electron. Mater. 2016, 45, 4010-4017. [CrossRef]

24. Davis, K.; Qi, B.; Witmer, M.; Kitchens, C.L.; Powell, B.A.; Mefford, O.T. Quantitative measurement of ligand exchange on iron oxides via radiolabeled oleic acid. Langmuir 2014, 30, 10918-10925. [CrossRef] [PubMed]

25. Majeed, M.I.; Lu, Q.; Yan, W.; Li, Z.; Hussain, I.; Tahir, M.N.; Tremel, W.; Tan, B. Highly water-soluble magnetic iron oxide $\left(\mathrm{Fe}_{3} \mathrm{O}_{4}\right)$ nanoparticles for drug delivery: Enhanced in vitro therapeutic efficacy of doxorubicin and mion conjugates. J. Mater. Chem. B 2013, 1, 2874-2884. [CrossRef]

26. Wang, H.; Shen, J.; Li, Y.; Wei, Z.; Cao, G.; Gai, Z.; Hong, K.; Banerjee, P.; Zhou, S. Magnetic iron oxide-fluorescent carbon dots integrated nanoparticles for dual-modal imaging, near-infrared light-responsive drug carrier and photothermal therapy. Biomater. Sci. 2014, 2, 915-923. [CrossRef]

27. Shi, D.; Sadat, M.E.; Dunn, A.W.; Mast, D.B. Photo-fluorescent and magnetic properties of iron oxide nanoparticles for biomedical applications. Nanoscale 2015, 7, 8209-8232. [CrossRef] [PubMed]

28. Hussain, I.; Graham, S.; Wang, Z.X.; Tan, B.; Sherrington, D.C.; Rannard, S.P.; Cooper, A.I.; Brust, M. Size-controlled synthesis of near-monodisperse gold nanoparticles in the $1-4 \mathrm{~nm}$ range using polymeric stabilizers. J. Am. Chem. Soc. 2005, 127, 16398-16399. [CrossRef] [PubMed]

29. Wang, Z.; Tan, B.; Hussain, I.; Schaeffer, N.; Wyatt, M.F.; Brust, M.; Cooper, A.I. Design of polymeric stabilizers for size-controlled synthesis of monodisperse gold nanoparticles in water. Langmuir 2007, 23, 885-895. [CrossRef] [PubMed]

30. Huang, X.; Luo, Y.; Li, Z.; Li, B.; Zhang, H.; Li, L.; Majeed, I.; Zou, P.; Tan, B. Biolabeling hematopoietic system cells using near-infrared fluorescent gold nanoclusters. J. Phys. Chem. C 2011, 115, 16753-16763. [CrossRef]

31. Schaeffer, N.; Tan, B.; Dickinson, C.; Rosseinsky, M.J.; Laromaine, A.; McComb, D.W.; Stevens, M.M.; Wang, Y.Q.; Petit, L.; Barentin, C.; et al. Fluorescent or not? Size-dependent fluorescence switching for polymer-stabilized gold clusters in the 1.1-1.7 nm size range. Chem. Commun. 2008, 34, 3986-3988. [CrossRef] [PubMed]

32. Liu, F.; Zhu, J.H.; Hou, Y.L.; Gao, S. Chemical synthesis of magnetic nanocrystals: Recent progress. Chin. Phys. B 2013, 22, 107503. [CrossRef]

33. Ito, D.; Yokoyama, S.; Zaikova, T.; Masuko, K.; Hutchison, J.E. Synthesis of ligand-stabilized metal oxide nanocrystals and epitaxial core/shell nanocrystals via a lower-temperature esterification process. ACS Nano 2014, 8, 64-75. [CrossRef] [PubMed]

34. Rui, Y.-P.; Liang, B.; Hu, F.; Xu, J.; Peng, Y.-F.; Yin, P.-H.; Duan, Y.; Zhang, C.; Gu, H. Ultra-large-scale production of ultrasmall superparamagnetic iron oxide nanoparticles for $\mathrm{t} 1$-weighted mri. RSC Adv. 2016, 6, 22575-22585. [CrossRef]

35. Wu, J.; Shen, Y.; Jiang, W.; Jiang, W.; Shen, Y. Magnetic targeted drug delivery carriers encapsulated with pH-sensitive polymer: Synthesis, characterization and in vitro doxorubicin release studies. J. Biomater. Sci. Polym. Ed. 2016, 27, 1303-1316. [CrossRef] [PubMed]

36. Zhuang, L.; Zhao, Y.; Zhong, H.; Liang, J.; Zhou, J.; Shen, H. Hydrophilic magnetochromatic nanoparticles with controllable sizes and super-high magnetization for visualization of magnetic field intensity. Sci. Rep. 2015, 5, 17063. [CrossRef] [PubMed]

37. Gupta, J.; Mohapatra, J.; Bhargava, P.; Bahadur, D. A pH-responsive folate conjugated magnetic nanoparticle for targeted chemo-thermal therapy and mri diagnosis. Dalton Trans. 2016, 45, 2454-2461. [CrossRef] [PubMed]

38. Hirt, A.M.; Sotiriou, G.A.; Kidambi, P.R.; Teleki, A. Effect of size, composition, and morphology on magnetic performance: First-order reversal curves evaluation of iron oxide nanoparticles. J. Appl. Phys. 2014, 115, 044314. [CrossRef]

39. Liu, G.; Gao, J.; Ai, H.; Chen, X. Applications and potential toxicity of magnetic iron oxide nanoparticles. Small 2013, 9, 1533-1545. [CrossRef] [PubMed]

(C) 2016 by the authors; licensee MDPI, Basel, Switzerland. This article is an open access article distributed under the terms and conditions of the Creative Commons Attribution (CC-BY) license (http://creativecommons.org/licenses/by/4.0/). 